\title{
Linkability of Some Blind Signature Schemes`
}

\author{
Swee-Huay Heng ${ }^{1}$, Wun-She Yap ${ }^{2}$, and Khoongming Khoo ${ }^{3}$ \\ ${ }^{1}$ Centre for Cryptography and Information Security (CCIS) \\ Faculty of Information Science and Technology \\ Multimedia University, Jalan Ayer Keroh Lama, 75450 Melaka, Malaysia \\ shheng@mmu .edu.my \\ ${ }^{2}$ Centre for Cryptography and Information Security (CCIS) \\ Faculty of Engineering \\ Multimedia University, 63100 Cyberjaya, Selangor, Malaysia \\ wsyap@mmu .edu.my \\ ${ }^{3}$ DSO National Laboratories \\ 20 Science Park Drive, Singapore 118230 \\ kkhoongm@dso.org.sg
}

\begin{abstract}
Unforgeability and blindness are two important properties of blind signature. The latter means that after interacting with various users, the signer is unable to link a valid message-signature pair. In ICCSA 2006, Zhang et al. showed that a signer in an identity-based blind signature scheme proposed by Huang et al. is able to link a valid messagesignature pair obtained by some user. They also presented an improved scheme to overcome this flaw. In ICICIC 2006, Zhang and Zou showed that the identity-based blind signature scheme proposed by Zhang and Kim also suffered from the similar linkability attack. In this paper, we first show that the so-called linkability can be shown for Zhang et al. scheme as well. We then point out that the linkability attack against the Huang et al. scheme and the Zhang-Kim scheme is invalid.
\end{abstract}

Keywords: Blind signature, identity-based, linkability, blindness.

\section{Introduction}

The concept of blind signatures was first introduced by Chaum [3] in 1982. A blind signature scheme is an interactive two-party protocol between a user and a signer. Informally, a blind signature is a signature scheme that incorporates a signing protocol that allows the signer to sign a document submitted by a user blindly, without obtaining any information about the document itself. This cryptographic scheme provides anonymity of users and is especially suited for use in e-cash and e-voting systems.

On the other hand, identity (ID)-based public key cryptography is a concept formalized by Shamir in 1984 [6]. In ID-based schemes, users need exchange

\footnotetext{
* The first and second authors gratefully acknowledge the Malaysia IRPA grant (0499-01-00003-EAR) and e-Science fund (01-02-01-SF0032).
} 
neither private keys nor public keys. Generally, an ID-based scheme is an asymmetric system wherein the public key is effectively replaced by or constructed from a user's publicly available identity information (e.g., name, email address, IP address) which uniquely identifies the user and can be undeniably associated with the user. The services of a trusted third party called private key generator $(\mathrm{PKG})$ are needed solely to generate private keys for users using the PKG's master-key and the user's public identity information. The main technical difference between ID-based cryptography and the traditional public key infrastructure (PKI) systems using certificates is in the binding between the public and private keys and the means of those keys. In a traditional PKI, this is achieved through the use of a certificate.

The first ID-based blind signature (IBBS) scheme was put forth by Zhang and Kim in 2002 [7. Later, the same authors provided an improved IBBS scheme 8. Unlike the first scheme, they claimed that the general parallel attack of this improved scheme does not depend on the difficulty of ROS-problem, this was then falsified by Huang et al. 4. Huang et al. showed that the security against generic parallel attack of Zhang and Kim's improved scheme 8] still depends on the difficulty of ROS-problem. Huang et al. 4] further proposed another scheme which offers advantages in runtime, communication and memory requirements over the first two schemes.

In ICCSA 2006, Zhang et al. showed that a signer in an IBBS scheme proposed by Huang et al. is able to link a valid message-signature pair obtained by some user [9]. They also presented an improved scheme to overcome this flaw. Recently, in ICICIC 2006, Zhang and Zou also showed that the identity-based blind signature scheme proposed by Zhang and Kim [7] is vulnerable to the same linkability attack [10]. In this paper, we first show that the so-called linkability can be shown for Zhang et al. scheme as well. We then show that the linkability attack is invalid. We also compare the performance between the Zhang-Kim scheme, the Zhang et al. scheme and the Huang et al. scheme. From the analysis, we can see that the Huang et al. scheme is more efficient than the Zhang et al. scheme.

In Section 2 we review some preliminaries. In Section 3, we review the Huang et al. and the Zhang-Kim IBBS scheme. In Section 4, we review the Zhang et al. scheme and discuss the linkability issue on the Zhang et al. scheme before falsifying the soundness of the linkability attack claimed by Zhang et al. against the Huang et al. scheme. Finally, we conclude this paper in Section 5.

\section{Preliminaries}

\subsection{Bilinear Pairings}

Throughout this paper, $\left(G_{1},+\right)$ and $\left(G_{2}, \cdot\right)$ denote two cyclic groups of prime order $q$. A bilinearmap, $e: G_{1} \times G_{1} \rightarrow G_{2}$ satisfies the following properties:

1. Bilinearity: For all $P, Q, R \in G_{1}, e(P+Q, R)=e(P, R) e(Q, R)$ and $e(P, Q+$ $R)=e(P, Q) e(P, R)$. 
2. Non-degeneracy: $e(P, Q) \neq 1$.

3. Computability: There is an efficient algorithm to compute $e(P, Q)$ for any $P, Q \in G_{1}$

\subsection{Identity-Based Blind Signature}

An identity-based blind signature (IBBS) scheme is considered as the combination of a general blind signature scheme and an ID-based one. In other words, it is a blind signature but the public key used in the verification is the signer identity such that no certificate is needed in authenticating the signer's public key. Now we review the framework and security model of an IBBS scheme [511784].

An IBBS scheme is a digital signature scheme which involves three parties: a trusted third party called the PKG, a signer and a user. It consists of the following four algorithms:

1. Setup is a probabilistic polynomial-time (PPT) algorithm run by the PKG that takes a security parameter $k$ and returns the system parameters params and master-key.

2. Extract is a deterministic algorithm run by the PKG that takes params, master-key and an entity identifier ID $\in\{0,1\}^{*}$ as input. It returns the signer private key $S_{I D}$.

3. Issue is an interactive PPT signature issuing protocol between a signer and a user. Suppose that the user is given its input tape (ID, $m$ ) where $m$ is a message and the signer is given its input tape (ID, $\left.S_{I D}\right)$. The signer and the user then engage in the signature issuing protocol. At the end of this protocol, the signer outputs either "completed" or "non-completed" while the user outputs either $\perp$ or the signature $\sigma$ of the message $m$.

4. Verify is a deterministic polynomial-time algorithm that accepts a signature $\sigma$, message $m$, params and ID and outputs true if the signature is correct, or $\perp$ otherwise.

These algorithms must satisfy the standard consistency constraint of an IDbased blind signature, i.e. if $\sigma=\operatorname{Issue}\left(m, \mathrm{ID}, S_{I D}, \operatorname{params}\right)$, Verify $(\sigma, m, \mathrm{ID}$, params $)=$ true must hold.

A secure ID-based blind signature should have the property of blindness and the unforgeability against adaptive chosen message and ID attacks. We provide the definition for the former only since we are particularly dealing with this notion in this paper.

Definition 1 (Blindness). Let $\mathcal{A}$ be the Signer or a PPT algorithm that controls the Signer. $\mathcal{A}$ is involved in the following game with two honest users, namely $U_{0}$ and $U_{1}$.

1. $\left(I D, S_{I D}\right) \leftarrow \operatorname{Extract}\left(\right.$ params,$\left.S_{I D}\right)$.

2. $\left(m_{0}, m_{1}\right) \leftarrow \mathcal{A}\left(I D, S_{I D}\right)$ ( $\mathcal{A}$ produces two messages).

3. Select $b \in\{0,1\}$. Put $m_{b}$ and $m_{1-b}$ to the read-only input tape of $U_{0}$ and $U_{1}$ respectively. 
4. $\mathcal{A}$ engages in the signature issuing protocol with $U_{0}$ and $U_{1}$ in an arbitrary order.

5. If $U_{0}$ and $U_{1}$ output $\sigma\left(m_{b}\right)$ and $\sigma\left(m_{1-b}\right)$ respectively using their private tapes, then give those outputs to $\mathcal{A}$. Otherwise, give $\perp$ to $\mathcal{A}$.

6. $\mathcal{A}$ outputs a bit $b^{\prime} \in\{0,1\}$.

We say that $\mathcal{A}$ wins the game if $b^{\prime}=b$. An IBBS is blind if there is no PPT algorithm $\mathcal{A}$ that wins the game with probability at least $1 / 2+1 / k^{c}$ for any constant $c>0$. The probability is taken over the coin flips of Extract, $U_{0}, U_{1}$ and $\mathcal{A}$.

\section{The Huang et al. and the Zhang-Kim IBBS Schemes}

\subsection{The Huang et al. IBBS Scheme}

1. Setup: Choose a group $G_{1}$ which is a cyclic additive group generated by $P$ with prime order $q$. Choose a cyclic multiplicative group $G_{2}$ with the same order $q$ and a bilinear pairing $e: G_{1} \times G_{1} \rightarrow G_{2}$. Pick a random $s \in Z_{q}^{*}$ and set $P_{p u b}=s P$. Choose two cryptographic hash functions $H_{1}$ : $\{0,1\}^{*} \times G_{2} \rightarrow Z_{q}^{*}$ and $H_{2}:\{0,1\}^{*} \rightarrow G_{1}$. Publicize the system parameters params $=\left(G_{1}, G_{2}, e, q, P, P_{p u b}, H_{1}, H_{2}\right)$ and keep the master key $s$ secret.

2. Extract: Given an identity ID, compute $P_{I D}=H_{2}(I D)$ and return the corresponding private key $S_{I D}=s P_{I D}$.

3. Issue: The user first chooses $P_{1} \in G_{1}$ and computes $e\left(P_{1}, P\right)$ beforehand. In order to get a signature on a message $m$, the interaction between the user and the signer is as follows:

- Sign (Part 1): The signer randomly chooses $r \in Z_{q}^{*}$ and computes $R^{\prime}=$ $e\left(P_{I D}, P_{\text {pub }}\right)^{r}$ before sending $R^{\prime}$ to the user as the commitment.

- Blinding: The user randomly chooses $t_{1}, t_{2} \in Z_{q}^{*}$ as blinding factors and computes $R=R^{\prime t_{1}} e\left(P_{1}, P\right)^{t_{2}}, h=H_{1}(m, R)$ and $h^{\prime}=h t_{1}$ before sending $h^{\prime}$ to the signer as the challenge.

- Sign (Part 2): The signer sends back $V^{\prime}$ to the user as the response where $V^{\prime}=\left(r h^{\prime}+1\right) S_{I D}$.

- Unblinding: The user checks whether $e\left(V^{\prime}, P\right)=R^{\prime h^{\prime}} e\left(P_{I D}, P_{p u b}\right)$. If yes, then the user computes $V=V^{\prime}+h t_{2} P_{1}$ and outputs the signature $\sigma=$ $(R, V)$.

4. Verify: To verify a signature $\sigma=(R, V)$ on a message $m$ for ID, the verifier checks whether $e(V, P)=R^{H_{1}(m, R)} e\left(P_{I D}, P_{p u b}\right)$.

\subsection{The Zhang-Kim IBBS Scheme}

1. Setup: The same as Section 3.1

2. Extract: The same as Section 3.1.

3. Issue:

- Sign (Part 1): The signer randomly chooses $r \in Z_{q}^{*}$ and computes $R=$ $r P$ before sending $R$ to the user as the commitment. 
- Blinding: The user randomly chooses $a, b \in Z_{q}^{*}$ as blinding factors and computes $t=e\left(b Q_{I D}+R+a P, P_{p u b}\right)$ and $c^{\prime}=H_{1}(m, t)+b$ before sending $c^{\prime}$ to the signer as the challenge.

- Sign (Part 2): The signer sends back $V^{\prime}$ to the user as the response where $V^{\prime}=c^{\prime} S_{I D}+r P_{p u b}$.

- Unblinding: The user computes $V=V^{\prime}+a P_{p u b}$ and $c=c^{\prime}-b$ and outputs the signature $\sigma=(V, c)$.

4. Verify: To verify a signature $\sigma=(V, c)$ on a message $m$ for ID, the verifier checks whether $c=H_{1}\left(m, e(V, P) e\left(Q_{I D}, P_{p u b}\right)^{-c}\right)$.

\section{Soundness of the Linkability Attack}

Recently, in ICCSA 2006, Zhang et al. claimed that the Huang et al. blind signature [4] did not satisfy the blindness by analyzing the security of the scheme where the signer is able to link a valid message-signature pair obtained by some user after interacting with various users 9]. In ICICIC 2006, Zhang and Zou also showed that the Zhang-Kim IBBS scheme [7] is vulnerable to the same linkability attack [10]. In this section, we first review the Zhang et al. scheme. We then review the Zhang et al. attack on the Huang et al. IBBS scheme and the Zhang-Zou attack on the Zhang-Kim IBBS scheme. Subsequently, we show that this so-called linkability attack can also be applied to the Zhang et al. scheme [9]. Finally, we prove that the so-called linkability attack is in fact invalid.

\subsection{The Zhang et al. IBBS Scheme}

The Zhang et al. scheme 9] is considered as an allegedly improved scheme over the Huang et al. which served as the countermeasure against the linkability attack mounted by Zhang et al. against the latter.

1. Setup: The same as Section 3.1

2. Extract: The same as Section 3.1

3. Issue: The user first chooses $P_{1} \in G_{1}$ and computes $e\left(P_{1}, P\right)$ beforehand. In order to get a signature on a message $m$, the interaction between the user and the signer is as follows:

- Sign (Part 1): The signer randomly chooses $r \in Z_{q}^{*}$ and computes $R^{\prime}=$ $e\left(P_{I D}, P_{\text {pub }}\right)^{r}$ before sending $R^{\prime}$ to the user as the commitment.

- Blinding: The user randomly chooses $t_{1}, t_{2}, t_{3} \in Z_{q}^{*}$ as the blinding factors and computes $R=R^{\prime t_{1}} e\left(P_{I D}, P_{p u b}\right)^{t_{1} t_{2}} e\left(P_{1}, P\right)^{t_{3}}, h=H_{1}(m, R)$ and $h^{\prime}=$ $h t_{1}^{-1}+t_{2}$ before sending $h^{\prime}$ to the signer as the challenge.

- Sign (Part 2): The signer sends back $V^{\prime}$ to the user as the response where $V^{\prime}=\left(r+h^{\prime}\right) S_{I D}$.

- Unblinding: The user computes $V=t_{1} V^{\prime}+t_{3} P_{1}$ and outputs the signature $\sigma=(R, V)$.

4. Verify: To verify a signature $\sigma=(R, V)$ on a message $m$ for ID, the verifier checks whether $e(V, P)=R \cdot e\left(P_{I D}, P_{p u b}\right)^{H_{1}(m, R)}$. 


\subsection{Linkability of the Huang et al. IBBS Scheme}

We briefly review the Zhang et al. attack below. During the interactive protocol execution between the signer and the user, the transcript $\left(R^{\prime}, h^{\prime}, V^{\prime}\right)$ is generated. Given a blind signature $\sigma=(R, V)$ on a message $m$, the signer executes the following steps:

1. Compute $\alpha=e\left(V-V^{\prime}, P\right)$.

2. Compute $\beta=R^{\prime h^{\prime}}$.

3. Compute $h=H_{1}(m, R)$ and check whether $\alpha \cdot \beta=R^{h}$. If equal, then it indicates that the signer is managed to link the message-signature pair.

Since $V=V^{\prime}+h t_{2} P_{1}$, thus the signer computes $\alpha$ as follows:

$$
\begin{aligned}
\alpha & =e\left(V-V^{\prime}, P\right) \\
& =e\left(h t_{2} P_{1}, P\right) \\
& =e\left(P_{1}, P\right)^{h t_{2}}
\end{aligned}
$$

The signer manages to compute $\beta=R^{\prime h^{\prime}}$ since $h^{\prime}=h t_{1}$ is known. Finally, $\alpha \cdot \beta$ is computed as follows:

$$
\begin{aligned}
\alpha \cdot \beta & =e\left(P_{1}, P\right)^{h t_{2}} \cdot R^{\prime h t_{1}} \\
& =\left\{e\left(P_{1}, P\right)^{t_{2}} \cdot R^{\prime t_{1}}\right\}^{h} \\
& \left.=R^{h} \text { where } R=R^{\prime t_{1}} e\left(P_{1}, P\right)^{t_{2}}\right)
\end{aligned}
$$

Thus, Zhang et al. claimed that the Huang et al. IBBS scheme 4 has no blindness.

\subsection{Linkability of the Zhang-Kim IBBS Scheme}

Zhang and Zou showed an attack on the Zhang-Kim IBBS scheme [7. We briefly review the Zhang and Zou attack now. During the interactive protocol execution between the signer and the user, the transcript $\left(R, c^{\prime}, V^{\prime}\right)$ is generated. Given a blind signature $\sigma=(c, V)$ on a message $m$, the signer executes the following steps:

1. Compute $\alpha=e\left(V-V^{\prime}, P\right)$.

2. Compute $\beta=c^{\prime}-c$.

3. Compute $\delta=e\left(R, P_{\text {pub }}\right)$.

4. Compute $t^{\prime}=\alpha \cdot \delta \cdot e\left(Q_{I D}, P_{p u b}\right)^{\beta}$.

5. Check whether $c=H_{1}\left(m, t^{\prime}\right)$.

Notice that

$$
\begin{aligned}
t^{\prime} & =\alpha \cdot \delta \cdot e\left(Q_{I D}, P_{p u b}\right)^{\beta} \\
& =e\left(V-V^{\prime}, P\right) \cdot e\left(R, P_{p u b}\right) \cdot e\left(Q_{I D}, P_{p u b}\right)^{\left(c^{\prime}-c\right)} \\
& =e\left(a P_{p u b}, P\right) \cdot e\left(R, P_{p u b}\right) \cdot e\left(Q_{I D}, P_{p u b}\right)^{b} \\
& =e\left(a P+R+b Q_{I D}, P_{p u b}\right) \\
& =t
\end{aligned}
$$


Thus, we have that the relation $H_{1}\left(m, t^{\prime}\right)=H_{1}(m, t)=c$ holds and it means that the signer is able to link a message-signature pair. Zhang and Zou then claimed that the Zhang-Kim IBBS scheme has no blindness as well.

\subsection{Linkability of the Zhang et al. Scheme}

Now, we show that the similar so-called linkability can be shown for the Zhang et al. scheme 9 as well.

During the interactive protocol execution between the signer and the user, the transcript $\left(R^{\prime}, h^{\prime}, V^{\prime}\right)$ is generated. Given a blind signature $\sigma=(R, V)$ on a message $m$, the signer executes the following steps:

1. Compute $\alpha=e\left(V-V^{\prime}, P\right)$.

2. compute $h=H_{1}(m, R)$ and set $\beta=h-h^{\prime}$.

3. Compute $t^{\prime}=\alpha \cdot e\left(P_{I D}, P_{p u b}\right)^{\beta} \cdot R^{\prime}$

4. Check whether $h=H\left(t^{\prime}, m\right)$. If equal, then it indicates that the signer is managed to link the message-signature pair.

Since $V=t_{1} V^{\prime}+t_{3} P_{1}$, the signer can compute $\alpha$ as follows:

$$
\begin{aligned}
\alpha & =e\left(V-V^{\prime}, P\right) \\
& =e\left(t_{1} V^{\prime}+t_{3} P_{1}-V^{\prime}, P\right) \\
& =e\left(\left(t_{1}-1\right) V^{\prime}+t_{3} P_{1}, P\right) \\
& =e\left(\left(t_{1}-1\right) V^{\prime}, P\right) e\left(t_{3} P_{1}, P\right) \\
& =e\left(\left(t_{1}-1\right)\left(r+h^{\prime}\right) S_{I D}, P\right) e\left(t_{3} P_{1}, P\right) \\
& =e\left(P_{I D}, P_{\text {pub }}\right)^{\left(t_{1}-1\right)\left(r+h^{\prime}\right)} e\left(P_{1}, P\right)^{t_{3}} \\
& =e\left(P_{I D}, P_{\text {pub }}\right)^{\left(r t_{1}-r+t_{1} h^{\prime}-h^{\prime}\right)} e\left(P_{1}, P\right)^{t_{3}}
\end{aligned}
$$

$\beta$ can be computed as $h^{\prime}-h$ since $h^{\prime}=h t_{1}^{-1}+t_{2}$ is known. Finally, $t^{\prime}$ is computed as follows:

$$
\begin{aligned}
t^{\prime} & =\alpha \cdot e\left(P_{I D}, P_{p u b}\right)^{\beta} \cdot R^{\prime} \\
& =e\left(P_{I D}, P_{\text {pub }}\right)^{\left(r t_{1}-r+t_{1} h^{\prime}-h^{\prime}\right)} e\left(P_{1}, P\right)^{t_{3}} e\left(P_{I D}, P_{p u b}\right)^{\beta} R^{\prime} \\
& =e\left(P_{I D}, P_{\text {pub }}\right)^{\left(r t_{1}-r+t_{1}\left(h t_{1}^{-1}+t_{2}\right)-h^{\prime}\right)} e\left(P_{1}, P\right)^{t_{3}} e\left(P_{I D}, P_{p u b}\right)^{h^{\prime}-h} e\left(P_{I D}, P_{p u b}\right)^{r} \\
& =e\left(P_{I D}, P_{\text {pub }}\right)^{\left(r t_{1}-r+h+t_{1} t_{2}-h^{\prime}\right)} e\left(P_{1}, P\right)^{t_{3}} e\left(P_{I D}, P_{\text {pub }}\right)^{h^{\prime}-h} e\left(P_{I D}, P_{p u b}\right)^{r} \\
& =e\left(P_{I D}, P_{\text {pub }}\right)^{\left(r t_{1}+t_{1} t_{2}\right)} e\left(P_{1}, P\right)^{t_{3}} \\
& =e\left(P_{I D}, P_{\text {pub }}\right)^{r t_{1}} e\left(P_{I D}, P_{\text {pub }}\right)^{t_{1} t_{2}} e\left(P_{1}, P\right)^{t_{3}} \\
& =R_{1}^{\prime t} e\left(P_{I D}, P_{p u b}\right)^{t_{1} t_{2}} e\left(P_{1}, P\right)^{t_{3}} \\
& =R
\end{aligned}
$$

Thus, we have $h=H_{1}\left(m, t^{\prime}\right)$. Assuming that the linkability attack shown by Zhang et al. and Zhang-Zhou is sound, then the Zhang et al. scheme which is an improvement over the Huang et al. scheme has no blindness too. 


\subsection{Soundness of the Linkability Attack}

At first glance, it seems that Zhang et al.'s claim is true. Nevertheless, we are going to show that their claim is wrong. The main reason is that this proposed attack works even if the blind signature is not generated from the protocol, meaning that even if there is totally no connection between the signature and the protocol transcript.

Let $\mathcal{A}$ be the signer or a PPT algorithm that controls the signer. $\mathcal{A}$ is involved in the blindness game with two honest users, namely $U_{0}$ and $U_{1}$. First, $b \in\{0,1\}$ is selected randomly. $\mathcal{A}$ engages in the Issue protocol with $U_{0}$ and $U_{1}$ in an arbitrary order. Assume that $U_{0}$ and $U_{1}$ output $\sigma\left(m_{b}\right)$ and $\sigma\left(m_{1-b}\right)$ respectively using their private tape, and give those outputs to $\mathcal{A}$. The output of the Issue protocol can be seen as in Table 1

Table 1. Output of the Issue Protocol

\begin{tabular}{|c||c|c|}
\hline & $U_{0}$ & $U_{1}$ \\
\hline Transcript & $\left(R_{0}^{\prime}, h_{0}^{\prime}, V_{0}^{\prime}\right)$ & $\left(R_{1}^{\prime}, h_{1}^{\prime}, V_{1}^{\prime}\right)$ \\
Resulting message-signature pair & $\left(m_{0}, R_{0}, V_{0}\right)$ & $\left(m_{1}, R_{1}, V_{1}\right)$ \\
\hline
\end{tabular}

Now, assume that $\mathcal{A}$ has the knowledge of $\left(R_{0}^{\prime}, h_{0}^{\prime}, V_{0}^{\prime}\right)$ and it wants to link the transcript with the output of $U_{1}: \sigma\left(m_{1}\right)=\left(R_{1}, V_{1}\right)$ in order to ensure the so-called linkability. We apply the Zhang et al. attack to show that the linkability algorithm always returns true even if the blind signature has totally no connection with the protocol transcript, thus we prove that $\mathcal{A}$ is unable to derive a link between a protocol view and a blind signature that has no relationship with the protocol view. This can be exhibited as follows:

1. Let $V_{0}^{\prime}=\left(r_{0} h_{0}^{\prime}+1\right) S_{I D}$ and $V_{1}=V_{1}^{\prime}+h_{1} t_{2} P_{1}=\left(r_{1} h_{1}^{\prime}+1\right) S_{I D}+h_{1} t_{2} P_{1}$.

2. Compute $\alpha$ as follows:

$$
\begin{aligned}
\alpha & =e\left(V_{1}-V_{0}^{\prime}, P\right) \\
& =e\left(\left\{\left(r_{1} h_{1}^{\prime}+1\right) S_{I D}+h_{1} t_{2} P_{1}\right\}-\left(r_{0} h_{0}^{\prime}+1\right) S_{I D}, P\right) \\
& =e\left(r_{1} h_{1}^{\prime} S_{I D}+h_{1} t_{2} P_{1}-r_{0} h_{0}^{\prime} S_{I D}, P\right) \\
& =e\left(\left(r_{1} h_{1}^{\prime}-r_{0} h_{0}^{\prime}\right) S_{I D}+h_{1} t_{2} P_{1}, P\right) \\
& =e\left(\left(r_{1} h_{1}^{\prime}-r_{0} h_{0}^{\prime}\right) S_{I D}, P\right) e\left(h_{1} t_{2} P_{1}, P\right) \\
& =e\left(P_{I D}, P_{p u b}\right)^{\left(r_{1} h_{1}^{\prime}-r_{0} h_{0}^{\prime}\right)} e\left(P_{1}, P\right)^{h_{1} t_{2}}
\end{aligned}
$$

3. Compute $\beta$ as follows:

$$
\beta=R_{0}^{\prime h_{0}^{\prime}}=e\left(P_{I D}, P_{p u b}\right)^{r_{0} h_{0}^{\prime}}
$$


4. Compute $\alpha \cdot \beta$ as follows:

$$
\begin{aligned}
\alpha \cdot \beta & =e\left(P_{I D}, P_{\text {pub }}\right)^{\left(r_{1} h_{1}^{\prime}-r_{0} h_{0}^{\prime}\right)} e\left(P_{1}, P\right)^{\left(h_{1} t_{2}\right)} \cdot e\left(P_{I D}, P_{p u b}\right)^{r_{0} h_{0}^{\prime}} \\
& =e\left(P_{I D}, P_{\text {pub }}\right)^{\left(r_{1} h_{1}^{\prime}-r_{0} h_{0}^{\prime}+r_{0} h_{0}^{\prime}\right)} e\left(P_{1}, P\right)^{h_{1} t_{2}} \\
& =e\left(P_{I D}, P_{p u b}\right)^{r_{1} h_{1} t_{1}} e\left(P_{1}, P\right)^{h_{1} t_{2}} \\
& =\left\{e\left(P_{I D}, P_{p u b}\right)^{r_{1} t_{1}} e\left(P_{1}, P\right)^{t_{2}}\right\}^{h_{1}} \\
& =R_{1}^{h_{1}}
\end{aligned}
$$

where $h_{1}^{\prime}=h_{1} t_{1}$ and $R_{1}=R_{1}^{\prime t_{1}} e\left(P_{1}, P\right)^{t_{2}}=e\left(P_{I D}, P_{p u b}\right)^{\left(r_{1} t_{1}\right)} e\left(P_{1}, P\right)^{t_{2}}$

Based on the above computation, the linking algorithm always returns true and thus this shows that $\left(R_{0}^{\prime}, h_{0}^{\prime}, V_{0}^{\prime}\right)$ can be linked with $\left(m_{1}, R_{1}, V_{1}\right)$. Hence, the proposed attack of Zhang et al. 9] is invalid. The similar analysis applies to the linkability attack on the Zhang-Kim scheme [7] and the Zhang et al. scheme 9.

\subsection{A Comparison}

We give a comparison between the Zhang-Kim scheme [7, the Huang et al. [4] and the Zhang et al. 9] IBBS schemes in terms of their computational complexity. We denote $B P$ as the bilinear pairing operation, $P M$ as the point multiplication on $G_{1}, P A$ as the point addition on $G_{1}$ and $E$ as the exponentiation on $G_{2}$. The result is summarized in Table 2

Table 2. A Comparison

\begin{tabular}{|c||c|c|}
\hline Scheme & Issue & Verify \\
\hline Zhang-Kim [7] & $1 B P+6 P M+4 P A$ & $2 B P+1 E$ \\
Huang et al. [4] & $2 P M+1 P A+4 E$ & $1 B P+1 E$ \\
Zhang et al. [9] & $3 P M+1 P A+4 E$ & $1 B P+1 E$ \\
\hline
\end{tabular}

It can be easily seen that the original Huang et al. scheme [4] is more efficient than the Zhang et al. scheme [9].

\section{Conclusion}

We falsified the linkability attack shown on the Huang et al. and the Zhang-Kim IBBS schemes by Zhang et al., and Zhang and Zou respectively. Thus, the claim that the Huang et al. and the Zhang-Kim schemes have no blindness is wrong. Besides, we also compared the efficiency of the Zhang-Kim scheme, Huang et al. scheme and the Zhang et al. scheme. Based on our analysis, the Huang et al. scheme is the most efficient scheme. 


\section{References}

1. M. Abe and T. Okamoto. Provably Secure Partially Blind Signature. In Proceedings of CRYPTO 2000, LNCS 1880, pp. 271-286, Springer-Verlag, 2000.

2. J. Cha and J. Cheon. An Identity-Based Signature from Gap Diffie-Hellman Groups. In Proceedings of PKC 2003, LNCS 2567, pp. 18-30, Springer-Verlag, 2003.

3. D. Chaum. Blind signatures for untraceable payments. In Proceedings of CRYPTO 1982, pp. 199-203, Springer-Verlag, 1983.

4. Z. Huang, K. Chen, Y. Wang. Efficient Identity-Based Signatures and Blind Signatures. In Proceedings of CANS 2005, LNCS 3574, pp. 120-133, Springer-Verlag, 2005.

5. D. Pointcheval and J. Stern. Provable Secure Blind Signature Schemes. In Proceedings of ASIACRYPT 1996, LNCS 1163, pp. 252-263, Springer-Verlag, 1996.

6. A. Shamir. Identity Based Cryptosystems and Signature Scheme. In Proceedings of CRYPTO 1984, LNCS 196, pp. 47-53, Springer-Verlag, 1984.

7. F. Zhang and K. Kim. ID-Based Blind Signature and Ring Signature from Pairings. In Proceedings of ASIACRYPT 2002, LNCS 2501, pp. 533-547, Springer-Verlag, 2002.

8. F. Zhang and K. Kim. Efficient ID-Based Blind Signature and Proxy Signature. In Proceedings of ACISP 2003, LNCS 2727, pp. 312-323, Springer-Verlag, 2003.

9. J. Zhang, T.Wei, J. Zhang and W. Zou. Linkability of a Blind Signature Scheme and Its Improved Scheme. In Proceedings of ICCSA 2006, LNCS 3983, pp. 262-270, Springer-Verlag, 2006.

10. J. Zhang and W. Zou. Linkability of a Blind Signature Scheme. In Proceedings of ICICIC 2006, Vol. 1, pp. 468-471, IEEE, 2006. 